\title{
Some Inclusion Relationships of Certain Subclasses of $p$-Valent Functions Associated with a Family of Integral Operators
}

\author{
M. K. Aouf, ${ }^{1}$ R. M. El-Ashwah, ${ }^{2}$ and Ahmed M. Abd-Eltawab ${ }^{3}$ \\ ${ }^{1}$ Department of Mathematics, Faculty of Science, Mansoura University, Mansoura 35516, Egypt \\ ${ }^{2}$ Department of Mathematics, Faculty of Science, Damietta University, New Damietta 34517, Egypt \\ ${ }^{3}$ Department of Mathematics, Faculty of Science, Fayoum University, Fayoum 63514, Egypt
}

Correspondence should be addressed to Ahmed M. Abd-Eltawab; ams03@fayoum.edu.eg

Received 16 June 2013; Accepted 24 August 2013

Academic Editors: G. Gripenberg and B. Wang

Copyright $(2013$ M. K. Aouf et al. This is an open access article distributed under the Creative Commons Attribution License, which permits unrestricted use, distribution, and reproduction in any medium, provided the original work is properly cited.

By making use of the new integral operator $\mathfrak{R}_{\beta, p}^{\alpha, \gamma}$, we introduce and investigate several new subclasses of $p$-valent starlike, $p$-valent convex, $p$-valent close-to-convex, and $p$-valent quasi-convex functions. In particular, we establish some inclusion relationships associated with the aforementioned integral operators. Some of the results established in this paper would provide extensions of those given in earlier works.

\section{Introduction}

Let $A(p)$ denote the class of functions of the form

$$
f(z)=z^{p}+\sum_{k=1}^{\infty} a_{k+p} z^{k+p} \quad(p \in \mathbb{N}=\{1,2,3, \ldots\})
$$

which are analytic and $p$-valent in the unit $\operatorname{disc} U=\{z: z \epsilon$ $\mathbb{C}$, and $|z|<1\}$ and let $A(1)=A$.

A function $f \in A(p)$ is said to be in the class $S_{p}^{*}(\lambda)$ of $p$-valent starlike functions of order $\lambda$ in $U$ if and only if

$$
\operatorname{Re}\left(\frac{z f^{\prime}(z)}{f(z)}\right)>\lambda \quad(z \in U ; 0 \leq \lambda<p) .
$$

The class $S_{p}^{*}(\lambda)$ was introduced by Patil and Thakare [1].

Owa [2] introduced the class $K_{p}(\lambda)$ of $p$-valent convex of order $\lambda$ in $U$ if and only if

$$
\operatorname{Re}\left(1+\frac{z f^{\prime \prime}(z)}{f^{\prime}(z)}\right)>\lambda \quad(z \in U ; 0 \leq \lambda<p) .
$$

It is easy to observe from (2) and (3) that

$$
f(z) \in K_{p}(\lambda) \Longleftrightarrow \frac{z f^{\prime}(z)}{p} \in S_{p}^{*}(\lambda) .
$$

We denote by $S_{p}^{*}=S_{p}^{*}(0)$ and $K_{p}=K_{p}(0)$ where $S_{p}^{*}$ and $K_{p}$ are the classes of $p$-valently starlike functions and $p$ valently convex functions, respectively, (see Goodman [3]).

For a function $f \in A(p)$, we say that $f \in C_{p}(\eta, \lambda)$ if there exists a function $g \in S_{p}^{*}(\lambda)$ such that

$$
\operatorname{Re}\left(\frac{z f^{\prime}(z)}{g(z)}\right)>\eta \quad(z \in U ; 0 \leq \lambda, \eta<p) .
$$

Functions in the class $C_{p}(\eta, \lambda)$ are called $p$-valent close-toconvex functions of order $\eta$ and type $\lambda$. The class $C_{p}(\eta, \lambda)$ was studied by Aouf [4] and the class $C_{1}(\eta, \lambda)$ was studied by Libera [5].

Noor $[6,7]$ introduced and studied the classes $C_{p}^{*}(\eta, \lambda)$ and $C_{1}^{*}(\eta, \lambda)$ as follows.

A function $f \in A(p)$ is said to be in the class $C_{p}^{*}(\eta, \lambda)$ of quasi-convex functions of order $\eta$ and type $\lambda$ if there exists a function $g \in K_{p}(\lambda)$ such that

$$
\operatorname{Re}\left\{\frac{\left(z f^{\prime}(z)\right)^{\prime}}{g^{\prime}(z)}\right\}>\eta \quad(z \in U ; 0 \leq \lambda, \quad \eta<p) .
$$


It follows from (5) and (6) that

$$
f(z) \in C_{p}^{*}(\eta, \lambda) \Longleftrightarrow \frac{z f^{\prime}(z)}{p} \in C_{p}(\eta, \lambda) .
$$
by

For functions $f \in A(p)$ given by (1) and $g \in A(p)$ given

$$
g(z)=z^{p}+\sum_{k=1}^{\infty} b_{k+p} z^{k+p} \quad(p \in \mathbb{N}),
$$

the Hadamard product (or convolution) of $f$ and $g$ is given by

$$
(f * g)(z)=z^{p}+\sum_{k=1}^{\infty} a_{k+p} b_{k+p} z^{k+p}=(g * f)(z) .
$$

For the function $f \in A(p)$, we introduced the operator $\mathfrak{R}_{\beta, p}^{\alpha, \gamma}: A(p) \rightarrow A(p)$ as follows:

$$
\begin{aligned}
\mathfrak{R}_{\beta, p}^{\alpha, \gamma} f(z)= & \left(\begin{array}{c}
p+\alpha+\beta-\gamma \\
p+\beta-1
\end{array}\right) \frac{(\alpha-\gamma+1)}{z^{\beta}} \\
& \times \int_{0}^{z}\left(1-\frac{t}{z}\right)^{\alpha-\gamma} t^{\beta-1} f(t) d t \\
= & \frac{\Gamma(p+\alpha+\beta-\gamma+1)}{\Gamma(p+\beta) \Gamma(\alpha-\gamma+1)} \frac{1}{z^{\beta}} \\
& \times \int_{0}^{z}\left(1-\frac{t}{z}\right)^{\alpha-\gamma} t^{\beta-1} f(t) d t \\
= & z^{p}+\frac{\Gamma(p+\alpha+\beta-\gamma+1)}{\Gamma(p+\beta)} \\
& \times \sum_{k=1}^{\infty}\left[\frac{\Gamma(\beta+p+k)}{\Gamma(\alpha+\beta+p+k-\gamma+1)}\right] a_{k+p} z^{k+p} \\
& (\beta>-p ; \alpha \geq \gamma-1 ; \gamma>0 ; p \in \mathbb{N} ; z \in U) .
\end{aligned}
$$

From (10), it is easy to verify that

$$
\begin{aligned}
z\left(\mathfrak{R}_{\beta, p}^{\alpha+1, \gamma} f(z)\right)^{\prime}= & (\alpha+\beta+p-\gamma+1) \mathfrak{R}_{\beta, p}^{\alpha, \gamma} f(z) \\
& -(\alpha+\beta-\gamma+1) \mathfrak{R}_{\beta, p}^{\alpha+1, \gamma} f(z) .
\end{aligned}
$$

Remark 1. Consider (i) For $\gamma=1$,

$$
\begin{aligned}
\mathfrak{R}_{\beta, p}^{\alpha, 1} f(z)= & Q_{\beta, p}^{\alpha} f(z) \\
= & \left(\begin{array}{c}
p+\alpha+\beta-1 \\
p+\beta-1
\end{array}\right) \frac{\alpha}{z^{\beta}} \int_{0}^{z}\left(1-\frac{t}{z}\right)^{\alpha-1} t^{\beta-1} f(t) d t \\
= & \frac{\Gamma(p+\alpha+\beta)}{\Gamma(p+\beta) \Gamma(\alpha)} \frac{1}{z^{\beta}} \int_{0}^{z}\left(1-\frac{t}{z}\right)^{\alpha-1} t^{\beta-1} f(t) d t \\
= & z^{p}+\frac{\Gamma(p+\alpha+\beta)}{\Gamma(p+\beta)} \\
& \times \sum_{k=1}^{\infty}\left[\frac{\Gamma(\beta+p+k)}{\Gamma(\alpha+\beta+p+k)}\right] a_{k+p} z^{k+p} \\
& \quad(\beta>-p ; \alpha \geq 0 ; p \in \mathbb{N} ; z \in U),
\end{aligned}
$$

where the operator $Q_{\beta, p}^{\alpha}$ was introduced and studied by Liu and Owa [8], and $Q_{\beta, 1}^{\alpha}=Q_{\beta}^{\alpha}$, where the operator $Q_{\beta}^{\alpha}$ was introduced and studied by Jung et al. [9];

(ii) For $\alpha=\gamma=1$ and $\beta=c$,

$$
\begin{array}{r}
\mathfrak{R}_{c, p}^{1,1} f(z)=J_{c, p}(z)=\frac{p+c}{z^{c}} \int_{0}^{z} t^{c-1} f(t) d t \\
=z^{p}+\sum_{k=1}^{\infty}\left(\frac{c+p}{c+k+p}\right) a_{k+p} z^{k+p} \\
(c>-p ; p \in \mathbb{N} ; z \in U),
\end{array}
$$

where $J_{c, p}(z)$ is the familiar integral operator, which was defined by Cho and $\operatorname{Kim}[10]$. The operator $J_{c, 1}(z)=J_{c}(z)$ was introduced by Bernardi [11] and we note that $J_{1,1}(z)=J(z)$ was introduced and studied by Libera [12] and Livingston [13].

The main object of this paper is to investigate the various inclusion relationships for each of the following subclasses of the normalized analytic function class $A(p)$ which are defined here by means of the operator $\Re_{\beta, p}^{\alpha, \gamma}$ given by (10).

Definition 2. In conjunction with (2) and (10),

$$
\begin{gathered}
S_{\alpha, \beta, \gamma, p}^{*}(\lambda)=\left\{f: f \in A(p), \mathfrak{R}_{\beta, p}^{\alpha, \gamma} f \in S_{p}^{*}(\lambda),\right. \\
0 \leq \lambda<p, p \in \mathbb{N}\} .
\end{gathered}
$$

Definition 3. In conjunction with (3) and (10),

$$
\begin{gathered}
K_{\alpha, \beta, \gamma, p}(\lambda)=\left\{f: f \in A(p), \mathfrak{R}_{\beta, p}^{\alpha, \gamma} f \in K_{p}(\lambda),\right. \\
0 \leq \lambda<p, p \in \mathbb{N}\} .
\end{gathered}
$$

Definition 4. In conjunction with (5) and (10),

$$
\begin{gathered}
C_{\alpha, \beta, \gamma, p}(\eta, \lambda)=\left\{f: f \in A(p), \mathfrak{R}_{\beta, p}^{\alpha, \gamma} f \in C_{p}(\eta, \lambda),\right. \\
0 \leq \lambda, \eta<p, p \in \mathbb{N}\} .
\end{gathered}
$$

Definition 5. In conjunction with (6) and (10),

$$
\begin{gathered}
C_{\alpha, \beta, \gamma, p}^{*}(\eta, \lambda)=\left\{f: f \in A(p), \Re_{\beta, p}^{\alpha, \gamma} f \in C_{p}^{*}(\eta, \lambda),\right. \\
0 \leq \lambda, \eta<p, p \in \mathbb{N}\} .
\end{gathered}
$$


Remark 6. Consider (I) For $\gamma=1$, in the above definitions, we have

$$
\begin{aligned}
& S_{\alpha, \beta, 1, p}^{*}(\lambda)=S_{\alpha, \beta, p}^{*}(\lambda) \\
& =\left\{f: f \in A(p), Q_{\beta, p}^{\alpha} f \in S_{p}^{*}(\lambda),\right. \\
& 0 \leq \lambda<p, p \in \mathbb{N}\} \\
& K_{\alpha, \beta, 1, p}(\lambda)=K_{\alpha, \beta, p}(\lambda) \\
& =\left\{f: f \in A(p), Q_{\beta, p}^{\alpha} f \in K_{p}(\lambda),\right. \\
& 0 \leq \lambda<p, p \in \mathbb{N}\} \text {, } \\
& C_{\alpha, \beta, 1, p}(\eta, \lambda)=C_{\alpha, \beta, p}(\eta, \lambda) \\
& =\left\{f: f \in A(p), Q_{\beta, p}^{\alpha} f \in C_{p}(\eta, \lambda),\right. \\
& 0 \leq \lambda, \eta<p, p \in \mathbb{N}\}, \\
& C_{\alpha, \beta, 1, p}^{*}(\eta, \lambda)=C_{\alpha, \beta, p}^{*}(\eta, \lambda) \\
& =\left\{f: f \in A(p), Q_{\beta, p}^{\alpha} f \in C_{p}^{*}(\eta, \lambda),\right. \\
& 0 \leq \lambda, \eta<p, p \in \mathbb{N}\} \text {. }
\end{aligned}
$$

(II) For $\alpha=\gamma=1$ and $\beta=c(c>-p)$, in the above definitions, we have

$$
\begin{aligned}
S_{1, c, 1, p}^{*}(\lambda)= & S_{c, p}^{*}(\lambda) \\
= & \left\{f: f \in A(p), J_{c, p} f(z) \in S_{p}^{*}(\lambda),\right. \\
& 0 \leq \lambda<p, p \in \mathbb{N}\}, \\
K_{1, c, 1, p}(\lambda)= & K_{c, p}(\lambda) \\
= & \left\{f: f \in A(p), J_{c, p} f(z) \in K_{p}(\lambda),\right. \\
& 0 \leq \lambda<p, p \in \mathbb{N}\}, \\
C_{1, c, 1, p}(\eta, \lambda)= & C_{c, p}(\eta, \lambda) \\
= & \left\{f: f \in A(p), J_{c, p} f(z) \in C_{p}(\eta, \lambda),\right. \\
& 0 \leq \lambda, \eta<p, p \in \mathbb{N}\}, \\
C_{1, c, 1, p}^{*}(\eta, \lambda)= & C_{c, p}^{*}(\eta, \lambda) \\
= & \left\{f: f \in A(p), J_{c, p} f(z) \in C_{p}^{*}(\eta, \lambda),\right. \\
& 0 \leq \lambda, \eta<p, p \in \mathbb{N}\} .
\end{aligned}
$$

(III) For $\gamma=p=1$, in the above definitions, we have

$$
\begin{aligned}
S_{\alpha, \beta, 1,1}^{*}(\lambda) & =S_{\alpha, \beta}^{*}(\lambda) \\
& =\left\{f: f \in A, Q_{\beta}^{\alpha} f \in S^{*}(\lambda), 0 \leq \lambda<1\right\}, \\
K_{\alpha, \beta, 1,1}(\lambda)= & K_{\alpha, \beta}(\lambda) \\
= & \left\{f: f \in A, Q_{\beta}^{\alpha} f \in K(\lambda), 0 \leq \lambda<1\right\}, \\
C_{\alpha, \beta, 1,1}(\eta, \lambda)= & C_{\alpha, \beta}(\eta, \lambda) \\
= & \left\{f: f \in A, Q_{\beta}^{\alpha} f \in C(\eta, \lambda), 0 \leq \lambda, \eta<1\right\}, \\
C_{\alpha, \beta, 1,1}^{*}(\eta, \lambda)= & C_{\alpha, \beta}^{*}(\eta, \lambda) \\
= & \left\{f: f \in A, Q_{\beta}^{\alpha} f \in C^{*}(\eta, \lambda),\right. \\
& 0 \leq \lambda, \eta<1\},
\end{aligned}
$$

where the classes $S_{\alpha, \beta}^{*}(\lambda), K_{\alpha, \beta}(\lambda), C_{\alpha, \beta}(\eta, \lambda)$, and $C_{\alpha, \beta}^{*}(\eta, \lambda)$ were introduced and studied by Gao et al. [14].

In order to establish our main results, we need the following lemma due to Miller and Mocanu [15].

Lemma 7 (see [15]). Let $\Theta$ be a complex-valued function such that

$\Theta: D \longrightarrow \mathbb{C}, \quad D \subset \mathbb{C} \times \mathbb{C} \quad(\mathbb{C}$ is the complex plane $)$

and let $u=u_{1}+i u_{2}, v=v_{1}+i v_{2}$. Suppose that $\Theta(u, v)$ satisfies the following conditions:

(i) $\Theta(u, v)$ is continuous in $D$;

(ii) $(1,0) \in D$ and $\operatorname{Re}\{\Theta(1,0)\}>0$;

(iii) $\operatorname{Re}\left\{\Theta\left(i u_{2}, v_{1}\right)\right\} \leq 0$ for all $\left(i u_{2}, v_{1}\right) \in D$ such that $v_{1} \leq$ $-(1 / 2)\left(1+u_{2}^{2}\right)$.

Let

$$
q(z)=1+q_{1} z+q_{2} z^{2}+\cdots
$$

be analytic in $U$ such that $\left(q(z), z q^{\prime}(z)\right) \in D$ for all $z \in U$. If

$$
\operatorname{Re}\left\{\Theta\left(q(z), z q^{\prime}(z)\right)\right\}>0 \quad(z \in U),
$$

then

$$
\operatorname{Re}\{q(z)\}>0 \quad(z \in U)
$$

\section{The Main Results}

In this section, we give several inclusion relationships for analytic function classes, which are associated with the integral operator $\mathfrak{R}_{\beta, p}^{\alpha, \gamma}$. Unless otherwise mentioned, we assume throughout this paper that $\beta>-p, \alpha \geq \gamma-1, \gamma>0, p \in \mathbb{N}$, and $z \in U$. 
Theorem 8. Let $0 \leq \lambda<p$. Then

$$
S_{\alpha, \beta, \gamma, p}^{*}(\lambda) \subset S_{\alpha+1, \beta, \gamma, p}^{*}(\lambda) \text {. }
$$

Proof. Let $f \in S_{\alpha, \beta, \gamma, p}^{*}(\lambda)$ and set

$$
\frac{z\left(\mathfrak{R}_{\beta, p}^{\alpha+1, \gamma} f(z)\right)^{\prime}}{\mathfrak{R}_{\beta, p}^{\alpha+1, \gamma} f(z)}-\lambda=(p-\lambda) q(z),
$$

where $q(z)$ is given by (22). By using identity (11), we obtain

$$
\frac{\mathfrak{R}_{\beta, p}^{\alpha, \gamma} f(z)}{\mathfrak{R}_{\beta, p}^{\alpha+1, \gamma} f(z)}=\frac{\lambda+(p-\lambda) q(z)+(\alpha+\beta-\gamma+1)}{\alpha+\beta+p-\gamma+1} .
$$

Differentiating (27) logarithmically with respect to $z$, we obtain

$$
\begin{aligned}
& \frac{z\left(\mathfrak{R}_{\beta, p}^{\alpha, \gamma} f(z)\right)^{\prime}}{\mathfrak{R}_{\beta, p}^{\alpha, \gamma} f(z)} \\
& \quad=\frac{z\left(\mathfrak{R}_{\beta, p}^{\alpha+1, \gamma} f(z)\right)^{\prime}}{\mathfrak{R}_{\beta, p}^{\alpha+1, \gamma} f(z)}+\frac{(p-\lambda) z q^{\prime}(z)}{(p-\lambda) q(z)+\lambda+\alpha+\beta-\gamma+1} \\
& =\lambda+(p-\lambda) q(z)+\frac{(p-\lambda) z q^{\prime}(z)}{(p-\lambda) q(z)+\lambda+\alpha+\beta-\gamma+1} .
\end{aligned}
$$

We now choose $u=q(z)=u_{1}+i u_{2}$ and $v=z q^{\prime}(z)=v_{1}+i v_{2}$, and define the function $\Theta$ by

$$
\Theta(u, v)=(p-\lambda) u+\frac{(p-\lambda) v}{(p-\lambda) u+\lambda+\alpha+\beta-\gamma+1} .
$$

Then, clearly $\Theta(u, v)$ satisfies the following conditions:

(i) $\Theta(u, v)$ is continuous in $D=(\mathbb{C} \backslash\{(\lambda+\alpha+\beta-\gamma+$ 1) $/(\lambda-p)\}) \times \mathbb{C}$

(ii) $(1,0) \in D$ and $\operatorname{Re}\{\Theta(1,0)\}=p-\lambda>0$;

(iii) for all $\left(i u_{2}, v_{1}\right) \in D$ such that $v_{1} \leq-(1 / 2)\left(1+u_{2}^{2}\right)$ we have

$$
\begin{aligned}
\operatorname{Re}\left\{\Theta\left(i u_{2}, v_{1}\right)\right\} & =\operatorname{Re}\left\{\frac{(p-\lambda) v_{1}}{(p-\lambda) i u_{2}+\lambda+\alpha+\beta-\gamma+1}\right\} \\
& =\frac{(p-\lambda)(\lambda+\alpha+\beta-\gamma+1) v_{1}}{(p-\lambda)^{2} u_{2}^{2}+(\lambda+\alpha+\beta-\gamma+1)^{2}} \\
& \leq-\frac{(p-\lambda)\left(1+u_{2}^{2}\right)(\lambda+\alpha+\beta-\gamma+1)}{2\left[(p-\lambda)^{2} u_{2}^{2}+(\lambda+\alpha+\beta-\gamma+1)^{2}\right]} \\
& <0,
\end{aligned}
$$

which shows that the function $\Theta$ satisfies the hypotheses of Lemma 7. Consequently, we easily obtain the inclusion relationship (25).
Theorem 9. Let $0 \leq \lambda<p, p \in \mathbb{N}$. Then

$$
K_{\alpha, \beta, \gamma, p}(\lambda) \subset K_{\alpha+1, \beta, \gamma, p}(\lambda) .
$$

Proof. Let $f \in K_{\alpha, \beta, \gamma, p}(\lambda)$. Then, from Definition 3, we have

$$
\mathfrak{R}_{\beta, p}^{\alpha, \gamma} f \in K_{p}(\lambda), \quad(0 \leq \lambda<p ; p \in \mathbb{N}) .
$$

Furthermore, in view of the relationship (4), we find that

$$
\frac{z}{p}\left(\Re_{\beta, p}^{\alpha, \gamma} f(z)\right)^{\prime} \in S_{p}^{*}(\lambda)
$$

that is, that

$$
\mathfrak{R}_{\beta, p}^{\alpha, \gamma}\left(\frac{z f^{\prime}(z)}{p}\right) \in S_{p}^{*}(\lambda)
$$

Thus, by using Definition 2 and Theorem 8, we have

$$
\frac{z f^{\prime}(z)}{p} \in S_{p}^{*}(\alpha, \beta, \gamma, \lambda) \subset S_{p}^{*}(\alpha+1, \beta, \gamma, \lambda)
$$

which implies that

$$
K_{\alpha, \beta, \gamma, p}(\lambda) \subset K_{\alpha+1, \beta, \gamma, p}(\lambda) .
$$

The proof of Theorem 9 is thus completed.

Theorem 10. Let $0 \leq \lambda, \eta<p$. Then

$$
C_{\alpha, \beta, \gamma, p}(\eta, \lambda) \subset C_{\alpha+1, \beta, \gamma, p}(\eta, \lambda) .
$$

Proof. Let $f \in C_{\alpha, \beta, \gamma, p}(\eta, \lambda)$. Then there exists a function $\Psi \in$ $S_{p}^{*}(\lambda)$ such that

$$
\operatorname{Re}\left\{\frac{z\left(\Re_{\beta, p}^{\alpha, \gamma} f(z)\right)^{\prime}}{\Psi(z)}\right\}>\eta \quad(0 \leq \lambda, \eta<p, z \in U) .
$$

We put

$$
\mathfrak{R}_{\beta, p}^{\alpha, \gamma} g(z)=\Psi(z)
$$

so that we have

$$
g \in S_{\alpha, \beta, \gamma, p}^{*}(\lambda), \quad \operatorname{Re}\left\{\frac{z\left(\mathfrak{R}_{\beta, p}^{\alpha, \gamma} f(z)\right)^{\prime}}{\mathfrak{R}_{\beta, p}^{\alpha, \gamma} g(z)}\right\}>\eta \quad(z \in U) .
$$

We next put

$$
\frac{z\left(\mathfrak{R}_{\beta, p}^{\alpha+1, \gamma} f(z)\right)^{\prime}}{\mathfrak{R}_{\beta, p}^{\alpha+1, \gamma} g(z)}=\eta+(p-\eta) q(z)
$$


where $q(z)$ is given by (22). Thus, by using identity (11), we obtain

$$
\begin{aligned}
& \frac{z\left(\mathfrak{R}_{\beta, p}^{\alpha, \gamma} f(z)\right)^{\prime}}{\mathfrak{R}_{\beta, p}^{\alpha, \gamma} \mathfrak{g}(z)}=\frac{\mathfrak{R}_{\beta, p}^{\alpha, \gamma}\left(z f^{\prime}(z)\right)}{\mathfrak{R}_{\beta, p}^{\alpha, \gamma} \mathfrak{g}(z)} \\
& =\left(z\left[\mathfrak{R}_{\beta, p}^{\alpha+1, \gamma}\left(z f^{\prime}(z)\right)\right]^{\prime}\right. \\
& \left.+(\alpha+\beta-\gamma+1) \Re_{\beta, p}^{\alpha+1, \gamma}\left(z f^{\prime}(z)\right)\right) \\
& \times\left(z\left[\Re_{\beta, p}^{\alpha+1, \gamma} g(z)\right]^{\prime}\right. \\
& \left.+(\alpha+\beta-\gamma+1) \mathfrak{R}_{\beta, p}^{\alpha+1, \gamma} \mathfrak{g}(z)\right)^{-1} \\
& =\left(\frac{z\left[\mathfrak{R}_{\beta, p}^{\alpha+1, \gamma}\left(z f^{\prime}(z)\right)\right]^{\prime}}{\mathfrak{R}_{\beta, p}^{\alpha+1, \gamma} g(z)}\right. \\
& \left.+(\alpha+\beta-\gamma+1) \frac{\mathfrak{R}_{\beta, p}^{\alpha+1, \gamma}\left(z f^{\prime}(z)\right)}{\mathfrak{R}_{\beta, p}^{\alpha+1, \gamma} g(z)}\right) \\
& \times\left(\frac{z\left[\mathfrak{R}_{\beta, p}^{\alpha+1, \gamma} g(z)\right]^{\prime}}{\mathfrak{R}_{\beta, p}^{\alpha+1, \gamma} \mathfrak{g}(z)}\right. \\
& +(\alpha+\beta-\gamma+1))^{-1} .
\end{aligned}
$$

Since $g \in S_{\alpha, \beta, \gamma, p}^{*}(\lambda)$, then from Theorem 8 we have $g \in$ $S_{\alpha+1, \beta, \gamma, p}^{*}(\lambda)$, so that we can put

$$
\frac{z\left[\mathfrak{R}_{\beta, p}^{\alpha+1, \gamma} g(z)\right]^{\prime}}{\mathfrak{R}_{\beta, p}^{\alpha+1, \gamma} \mathfrak{g}(z)}=\lambda+(p-\lambda) G(z),
$$

where

$$
\begin{aligned}
& G(z)=g_{1}(x, y)+i g_{2}(x, y), \\
& \operatorname{Re}(G(z))=g_{1}(x, y)>0 \quad(z \in U) .
\end{aligned}
$$

Then

$$
\begin{aligned}
\frac{z\left(\mathfrak{R}_{\beta, p}^{\alpha, \gamma} f(z)\right)^{\prime}}{\mathfrak{R}_{\beta, p}^{\alpha, \gamma} g(z)}= & \left(\frac{z\left[\mathfrak{R}_{\beta, p}^{\alpha+1, \gamma}\left(z f^{\prime}(z)\right)\right]^{\prime}}{\mathfrak{R}_{\beta, p}^{\alpha+1, \gamma} g(z)}\right. \\
& +(\alpha+\beta-\gamma+1)(\eta+(p-\eta) q(z))) \\
& \times((p-\lambda) G(z)+\lambda+\alpha+\beta-\gamma+1)^{-1} .
\end{aligned}
$$

We thus find from (41) that

$$
z\left(\mathfrak{R}_{\beta, p}^{\alpha+1, \gamma} f(z)\right)^{\prime}=\mathfrak{R}_{\beta, p}^{\alpha+1, \gamma} g(z)[\eta+(p-\eta) q(z)] .
$$

Differentiating both sides of (46) with respect to $z$, we obtain

$$
\begin{aligned}
& \frac{z\left[z\left(\Re_{\beta, p}^{\alpha+1, \gamma} f(z)\right)^{\prime}\right]^{\prime}}{\mathfrak{R}_{\beta, p}^{\alpha+1, \gamma} g(z)} \\
& \quad=(p-\eta) z q^{\prime}(z)+\frac{z\left[\mathfrak{R}_{\beta, p}^{\alpha+1, \gamma} g(z)\right]^{\prime}}{\mathfrak{R}_{\beta, p}^{\alpha+1, \gamma} g(z)}[\eta+(p-\eta) q(z)] \\
& =(p-\eta) z q^{\prime}(z) \\
& \quad+[\lambda+(p-\lambda) G(z)][\eta+(p-\eta) q(z)] .
\end{aligned}
$$

By substituting (47) into (45), we have

$$
\begin{aligned}
\frac{z\left(\mathfrak{R}_{\beta, p}^{\alpha, \gamma} f(z)\right)^{\prime}}{\mathfrak{R}_{\beta, p}^{\alpha, \gamma} g(z)}-\eta= & (p-\eta) q(z) \\
& +\frac{(p-\eta) z q^{\prime}(z)}{(p-\lambda) G(z)+\lambda+\alpha+\beta-\gamma+1} .
\end{aligned}
$$

The remainder of our proof of Theorem 10 is much akin to that of Theorem 8 . We, therefore, choose to omit the details involved.

Theorem 11. Let $0 \leq \lambda, \eta<p$. Then

$$
C_{\alpha, \beta, \gamma, p}^{*}(\eta, \lambda) \subset C_{\alpha+1, \beta, \gamma, p}^{*}(\eta, \lambda) .
$$

Proof. Just as we derived Theorem 9 as a consequence of Theorem 8 by using the equivalence (4), we can also prove Theorem 11 by using Theorem 10 in conjunction with the equivalence (7).

Our main results in Theorems 8-11, can thus be applied with a view to deducing the following corollaries.

Taking $\gamma=1$ in Theorems 8-11 above, we obtain the following corollary.

Corollary 12. Let $0 \leq \lambda, \eta<p$. Then

$$
\begin{gathered}
S_{\alpha, \beta, p}^{*}(\lambda) \subset S_{\alpha+1, \beta, p}^{*}(\lambda), \\
K_{\alpha, \beta, p}(\lambda) \subset K_{\alpha+1, \beta, p}(\lambda), \\
C_{\alpha, \beta, p}(\eta, \lambda) \subset C_{\alpha+1, \beta, p}(\eta, \lambda), \\
C_{\alpha, \beta, p}^{*}(\eta, \lambda) \subset C_{\alpha+1, \beta, p}^{*}(\eta, \lambda) .
\end{gathered}
$$

Remark 13. Taking $p=1$ in Corollary 12, we obtain the results obtained by Gao et al. [14, Theorems 1-4].

Taking $\alpha=\gamma=1$ and $\beta=c(c>-p)$ in Theorems 8-11, we obtain the following corollary. 
Corollary 14. Let $0 \leq \lambda, \eta<p$. Then

$$
\begin{gathered}
S_{1, c, p}^{*}(\lambda) \subset S_{2, c, p}^{*}(\lambda), \\
K_{1, c, p}(\lambda) \subset K_{2, c, p}(\lambda), \\
C_{1, c, p}(\eta, \lambda) \subset C_{2, c, p}(\eta, \lambda), \\
C_{1, c, p}^{*}(\eta, \lambda) \subset C_{2, c, p}^{*}(\eta, \lambda) .
\end{gathered}
$$

Remark 15. Taking $p=1$ in Corollary 14, we obtain the results obtained by Gao et al. [14, Corollary 1-4].

\section{A Set of Integral-Preserving Properties}

In this section, we present several integral-preserving properties of the analytic function classes introduced here. In order to obtain the integral-preserving properties involving the integral operator $J_{c, p}$ defined by (13).

Theorem 16. Let $c$ be any real number and $c>-p$. If $f(z) \in$ $S_{\alpha, \beta, \gamma, p}^{*}(\lambda)$, then $J_{c, p}(z) \in S_{\alpha, \beta, \gamma, p}^{*}(\lambda)$, where $J_{c, p} f(z)$ is defined by (13).

Proof. From (13), we have

$$
z\left(\Re_{\beta, p}^{\alpha, \gamma} J_{c, p}(z)\right)^{\prime}=(c+p) \Re_{\beta, p}^{\alpha, \gamma}-c \Re_{\beta, p}^{\alpha, \gamma} J_{c, p}(z) .
$$

Let $f \in S_{\alpha, \beta, \gamma, p}^{*}(\lambda)$ and set

$$
\frac{z\left(\mathfrak{R}_{\beta, p}^{\alpha, \gamma} J_{c, p} f(z)\right)^{\prime}}{\mathfrak{R}_{\beta, p}^{\alpha, \gamma} J_{c, p} f(z)}-\lambda=(p-\lambda) q(z),
$$

where $q(z)$ is given by (22). By using identity (52), we obtain

$$
\frac{\mathfrak{R}_{\beta, p}^{\alpha, \gamma} f(z)}{\mathfrak{R}_{\beta, p}^{\alpha, \gamma} J_{c, p} f(z)}=\frac{\lambda+(p-\lambda) q(z)+c}{c+p} .
$$

Differentiating (54) logarithmically with respect to $z$, we obtain

$$
\begin{aligned}
\frac{z\left(\mathfrak{R}_{\beta, p}^{\alpha, \gamma} f(z)\right)^{\prime}}{\mathfrak{R}_{\beta, p}^{\alpha, \gamma} f(z)}= & \frac{z\left(\mathfrak{R}_{\beta, p}^{\alpha, \gamma} J_{c, p} f(z)\right)^{\prime}}{\mathfrak{R}_{\beta, p}^{\alpha, \gamma} J_{c, p} f(z)} \\
& +\frac{(p-\lambda) z q^{\prime}(z)}{(p-\lambda) q(z)+\lambda+c} \\
= & \lambda+(p-\lambda) q(z)+\frac{(p-\lambda) z q^{\prime}(z)}{(p-\lambda) q(z)+\lambda+c} .
\end{aligned}
$$

We now choose $u=q(z)=u_{1}+i u_{2}$, and $v=z q^{\prime}(z)=v_{1}+i v_{2}$, and define the function $\Theta$ by

$$
\Theta(u, v)=(p-\lambda) u+\frac{(p-\lambda) v}{(p-\lambda) u+\lambda+c} .
$$

It is easy to see that the function $\Theta(u, v)$ satisfies the conditions of Lemma 7 , and the remaining part of the proof of Theorem 16 is similar to that of Theorem 8 .
Taking $\gamma=1$ in Theorem 16, we obtain the following corollary.

Corollary 17. Let $c$ be any real number and $c>-p$. If $f(z) \in$ $S_{\alpha, \beta, p}^{*}(\lambda)$, then $J_{c, p}(z) \in S_{\alpha, \beta, p}^{*}(\lambda)$, where $J_{c, p} f(z)$ is defined by (13).

Theorem 18. Let $c$ be any real number and $c>-p$. If $f(z) \in$ $K_{\alpha, \beta, \gamma, p}(\lambda)$, then $J_{c, p}(z) \in K_{\alpha, \beta, \gamma, p}(\lambda)$, where $J_{c, p} f(z)$ is defined by (13).

Proof. By applying Theorem 16 in conjunction with the equivalence (4), it follows that

$$
\begin{aligned}
f(z) & \in K_{\alpha, \beta, \gamma, p}(\lambda) \\
& \Longleftrightarrow \frac{z}{p} f^{\prime}(z) \in S_{\alpha, \beta, \gamma, p}^{*}(\lambda) \\
& \Longrightarrow J_{c, p}\left(\frac{z}{p} f^{\prime}(z)\right) \in S_{\alpha, \beta, \gamma, p}^{*}(\lambda) \\
& \Longleftrightarrow \frac{z}{p}\left(J_{c, p} f(z)\right)^{\prime} \in S_{\alpha, \beta, \gamma, p}^{*}(\lambda) \\
& \Longrightarrow J_{c, p} f(z) \in K_{\alpha, \beta, \gamma, p}(\lambda),
\end{aligned}
$$

which proves Theorem 18

Taking $\gamma=1$ in Theorem 18, we obtain the following corollary.

Corollary 19. Let $c$ be any real number and $c>-p$. If $f(z) \in$ $K_{\alpha, \beta, p}(\lambda)$, then $J_{c, p}(z) \in K_{\alpha, \beta, p}(\lambda)$, where $J_{c, p} f(z)$ is defined by (13).

Theorem 20. Let $c$ be any real number and $c>-p$. If $f(z) \in$ $C_{\alpha, \beta, \gamma, p}(\eta, \lambda)$, then $J_{c, p}(z) \in C_{\alpha, \beta, \gamma, p}(\eta, \lambda)$, where $J_{c, p} f(z)$ is defined by (13).

Proof. Let $f \in C_{\alpha, \beta, \gamma, p}(\eta, \lambda)$. Then there exists a function $\Psi \in$ $S_{p}^{*}(\lambda)$ such that

$$
\operatorname{Re}\left\{\frac{z\left(\mathfrak{R}_{\beta, p}^{\alpha, \gamma} f(z)\right)^{\prime}}{\Psi(z)}\right\}>\eta \quad(0 \leq \lambda, \eta<p, z \in U) .
$$

We put

$$
\mathfrak{R}_{\beta, p}^{\alpha, \gamma} \mathfrak{g}(z)=\Psi(z)
$$

so that we have

$$
g \in S_{\alpha, \beta, \gamma, p}^{*}(\lambda), \quad \operatorname{Re}\left\{\frac{z\left(\mathfrak{R}_{\beta, p}^{\alpha, \gamma} f(z)\right)^{\prime}}{\mathfrak{R}_{\beta, p}^{\alpha, \gamma} g(z)}\right\}>\eta \quad(z \in U) .
$$

We next put

$$
\frac{z\left(\mathfrak{R}_{\beta, p}^{\alpha, \gamma} J_{c, p} f(z)\right)^{\prime}}{\mathfrak{R}_{\beta, p}^{\alpha, \gamma} J_{c, p} g(z)}=\eta+(p-\eta) q(z),
$$


where $q(z)$ is given by (22). Thus, by using identity (52), we obtain

$$
\begin{aligned}
& \frac{z\left(\mathfrak{R}_{\beta, p}^{\alpha, \gamma} J_{c, p} f(z)\right)^{\prime}}{\mathfrak{R}_{\beta, p}^{\alpha, \gamma} J_{c, p} \mathcal{g}(z)}=\frac{\mathfrak{R}_{\beta, p}^{\alpha, \gamma}\left(z\left(J_{c, p} f(z)\right)^{\prime}\right)}{\mathfrak{R}_{\beta, p}^{\alpha, \gamma} J_{c, p} \mathcal{g}(z)} \\
& =\left(z\left[\Re_{\beta, p}^{\alpha, \gamma}\left(z\left(J_{c, p} f(z)\right)^{\prime}\right)\right]^{\prime}\right. \\
& \left.+c \Re_{\beta, p}^{\alpha, \gamma}\left(z\left(J_{c, p} f(z)\right)^{\prime}\right)\right) \\
& \times\left(z\left[\mathfrak{R}_{\beta, p}^{\alpha, \gamma} J_{c, p} \mathcal{G}(z)\right]^{\prime}\right. \\
& \left.+c \Re_{\beta, p}^{\alpha, \gamma} J_{c, p} g(z)\right)^{-1} \\
& =\left(\frac{z\left[\mathfrak{R}_{\beta, p}^{\alpha, \gamma}\left(z\left(J_{c, p} f(z)\right)^{\prime}\right)\right]^{\prime}}{\mathfrak{R}_{\beta, p}^{\alpha, \gamma} J_{c, p} \mathcal{G}(z)}\right. \\
& \left.+c \frac{\mathfrak{R}_{\beta, p}^{\alpha, \gamma}\left(z\left(J_{c, p} f(z)\right)^{\prime}\right)}{\mathfrak{R}_{\beta, p}^{\alpha, \gamma} J_{c, p} g(z)}\right) \\
& \times\left(\frac{z\left[\mathfrak{R}_{\beta, p}^{\alpha, \gamma} J_{c, p} g(z)\right]^{\prime}}{\mathfrak{R}_{\beta, p}^{\alpha, \gamma} J_{c, p} g(z)}+c\right)^{-1} .
\end{aligned}
$$

Since $g \in S_{\alpha, \beta, \gamma, p}^{*}(\lambda)$, then from Theorem 16 we have $J_{c, p} g(z) \in S_{\alpha, \beta, \gamma, p}^{*}(\lambda)$, so that we can put

$$
\frac{z\left[\mathfrak{R}_{\beta, p}^{\alpha, \gamma} J_{c, p} \mathcal{g}(z)\right]^{\prime}}{\mathfrak{R}_{\beta, p}^{\alpha, \gamma} J_{c, p} \mathcal{G}(z)}=\lambda+(p-\lambda) G(z),
$$

where

$$
\begin{array}{r}
G(z)=g_{1}(x, y)+i g_{2}(x, y), \quad \operatorname{Re}(G(z))=g_{1}(x, y)>0 \\
(z \in U) .
\end{array}
$$

Then

$$
\begin{aligned}
\frac{z\left(\mathfrak{R}_{\beta, p}^{\alpha, \gamma} J_{c, p} f(z)\right)^{\prime}}{\mathfrak{R}_{\beta, p}^{\alpha, \gamma} J_{c, p} g(z)}= & \left(\frac{z\left[\mathfrak{R}_{\beta, p}^{\alpha, \gamma}\left(z\left(J_{c, p} f(z)\right)^{\prime}\right)\right]^{\prime}}{\mathfrak{R}_{\beta, p}^{\alpha, \gamma} J_{c, p} g(z)}\right. \\
& +c(\eta+(p-\eta) q(z))) \\
& \times((p-\lambda) G(z)+\lambda+c)^{-1} .
\end{aligned}
$$

We thus find from (61) that

$$
z\left(\mathfrak{R}_{\beta, p}^{\alpha, \gamma} J_{c, p} f(z)\right)^{\prime}=\mathfrak{R}_{\beta, p}^{\alpha, \gamma} J_{c, p} g(z)[\eta+(p-\eta) q(z)] .
$$

Differentiating both sides of (66) with respect to $z$, we obtain

$$
\begin{aligned}
\frac{z\left[z\left(\Re_{\beta, p}^{\alpha, \gamma} J_{c, p} f(z)\right)^{\prime}\right]^{\prime}}{\mathfrak{R}_{\beta, p}^{\alpha, \gamma} J_{c, p} g(z)} \\
=(p-\eta) z q^{\prime}(z) \\
\quad+\frac{z\left[\Re_{\beta, p}^{\alpha, \gamma} J_{c, p} g(z)\right]^{\prime}}{\mathfrak{R}_{\beta, p}^{\alpha, \gamma} J_{c, p} g(z)}[\eta+(p-\eta) q(z)] \\
=(p-\eta) z q^{\prime}(z) \\
\quad+[\lambda+(p-\lambda) G(z)][\eta+(p-\eta) q(z)] .
\end{aligned}
$$

By substituting (67) into (65), we have

$$
\frac{z\left(\Re_{\beta, p}^{\alpha, \gamma} f(z)\right)^{\prime}}{\mathfrak{R}_{\beta, p}^{\alpha, \gamma} g(z)}-\eta=(p-\eta) q(z)+\frac{(p-\eta) z q^{\prime}(z)}{(p-\lambda) G(z)+\lambda+c} .
$$

The remainder of our proof of Theorem 20 is much akin to that of Theorem 8 . We, therefore, choose to omit the details involved.

Taking $\gamma=1$ in Theorem 20, we obtain the following corollary.

Corollary 21. Let $c$ be any real number and $c>-p$. If $f(z) \in C_{\alpha, \beta, p}(\eta, \lambda)$, then $J_{c, p}(z) \in C_{\alpha, \beta, p}(\eta, \lambda)$, where $J_{c, p} f(z)$ is defined by (13).

Theorem 22. Let $c$ be any real number and $c>-p$. If $f(z) \in$ $C_{\alpha, \beta, \gamma, p}^{*}(\eta, \lambda)$, then $J_{c, p}(z) \in C_{\alpha, \beta, \gamma, p}^{*}(\eta, \lambda)$, where $J_{c, p} f(z)$ is defined by (13).

Proof. Just as we derived Theorem 18 as a consequence of Theorem 16 by using the equivalence (4), we can also prove Theorem 22 by using Theorem 20 in conjunction with the equivalence (7).

Taking $\gamma=1$ in Theorem 22, we obtain the following corollary.

Corollary 23. Let $c$ be any real number and $c>-p$. If $f(z) \in$ $C_{\alpha, \beta, \gamma, p}^{*}(\eta, \lambda)$, then $J_{c, p}(z) \in C_{\alpha, \beta, \gamma, p}^{*}(\eta, \lambda)$, where $J_{c, p} f(z)$ is defined by (13).

\section{References}

[1] D. A. Patil and N. K. Thakare, "On convex hulls and extreme points of $p$-valent starlike and convex classes with applications," Bulletin Mathématique de la Société des Sciences Mathématiques de la République Socialiste de Roumanie, vol. 27, no. 75, pp. 145160,1983

[2] S. Owa, "On certain classes of $p$-valent functions with negative coefficients," Simon Stevin. A Quarterly Journal of Pure and Applied Mathematics, vol. 59, no. 4, pp. 385-402, 1985. 
[3] A. W. Goodman, "On the Schwarz-Christoffel transformation and p-valent functions," Transactions of the American Mathematical Society, vol. 68, pp. 204-223, 1950.

[4] M. K. Aouf, "On a class of $p$-valent close-to-convex functions of order $\beta$ and type $\alpha$," International Journal of Mathematics and Mathematical Sciences, vol. 11, no. 2, pp. 259-266, 1988.

[5] R. J. Libera, "Some radius of convexity problems," Duke Mathematical Journal, vol. 31, pp. 143-158, 1964.

[6] K. I. Noor, "On quasi-convex functions and related topics," International Journal of Mathematics and Mathematical Sciences, vol. 10, no. 2, pp. 241-258, 1987.

[7] K. I. Noor, "On some applications of the Ruscheweyh derivatives," Mathematica Japonica, vol. 36, no. 5, pp. 869-874, 1991.

[8] J.-L. Liu and S. Owa, "Properties of certain integral operator," International Journal of Mathematical Sciences, vol. 3, no. 1, pp. 69-75, 2004.

[9] I. B. Jung, Y. C. Kim, and H. M. Srivastava, "The Hardy space of analytic functions associated with certain one-parameter families of integral operators," Journal of Mathematical Analysis and Applications, vol. 176, no. 1, pp. 138-147, 1993.

[10] N. E. Cho and T. H. Kim, "Multiplier transformations and strongly close-to-convex functions," Bulletin of the Korean Mathematical Society, vol. 40, no. 3, pp. 399-410, 2003.

[11] S. D. Bernardi, "Convex and starlike univalent functions," Transactions of the American Mathematical Society, vol. 135, pp. 429-446, 1969.

[12] R. J. Libera, "Some classes of regular univalent functions," Proceedings of the American Mathematical Society, vol. 16, pp. 755-758, 1965.

[13] A. E. Livingston, "On the radius of univalence of certain analytic functions," Proceedings of the American Mathematical Society, vol. 17, pp. 352-357, 1966.

[14] C.-Y. Gao, S.-M. Yuan, and H. M. Srivastava, "Some functional inequalities and inclusion relationships associated with certain families of integral operators," Journal of Computational and Applied Mathematics, vol. 49, no. 11-12, pp. 1787-1795, 2005.

[15] S. S. Miller and P. T. Mocanu, "Second-order differential inequalities in the complex plane," Journal of Mathematical Analysis and Applications, vol. 65, no. 2, pp. 289-305, 1978. 


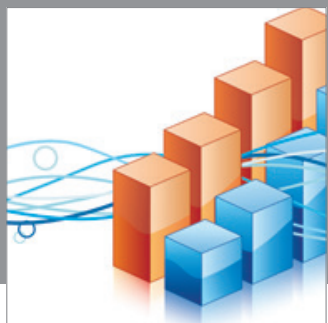

Advances in

Operations Research

mansans

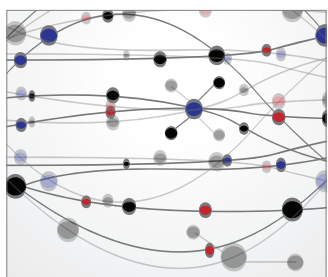

The Scientific World Journal
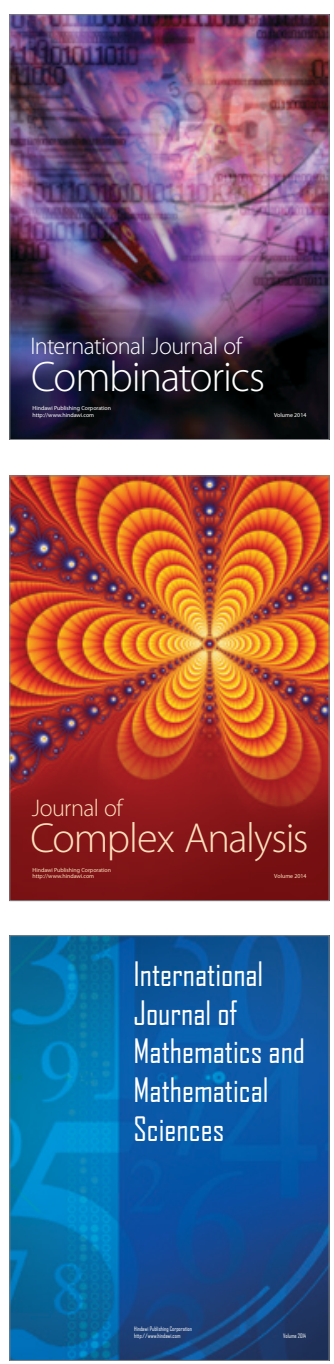
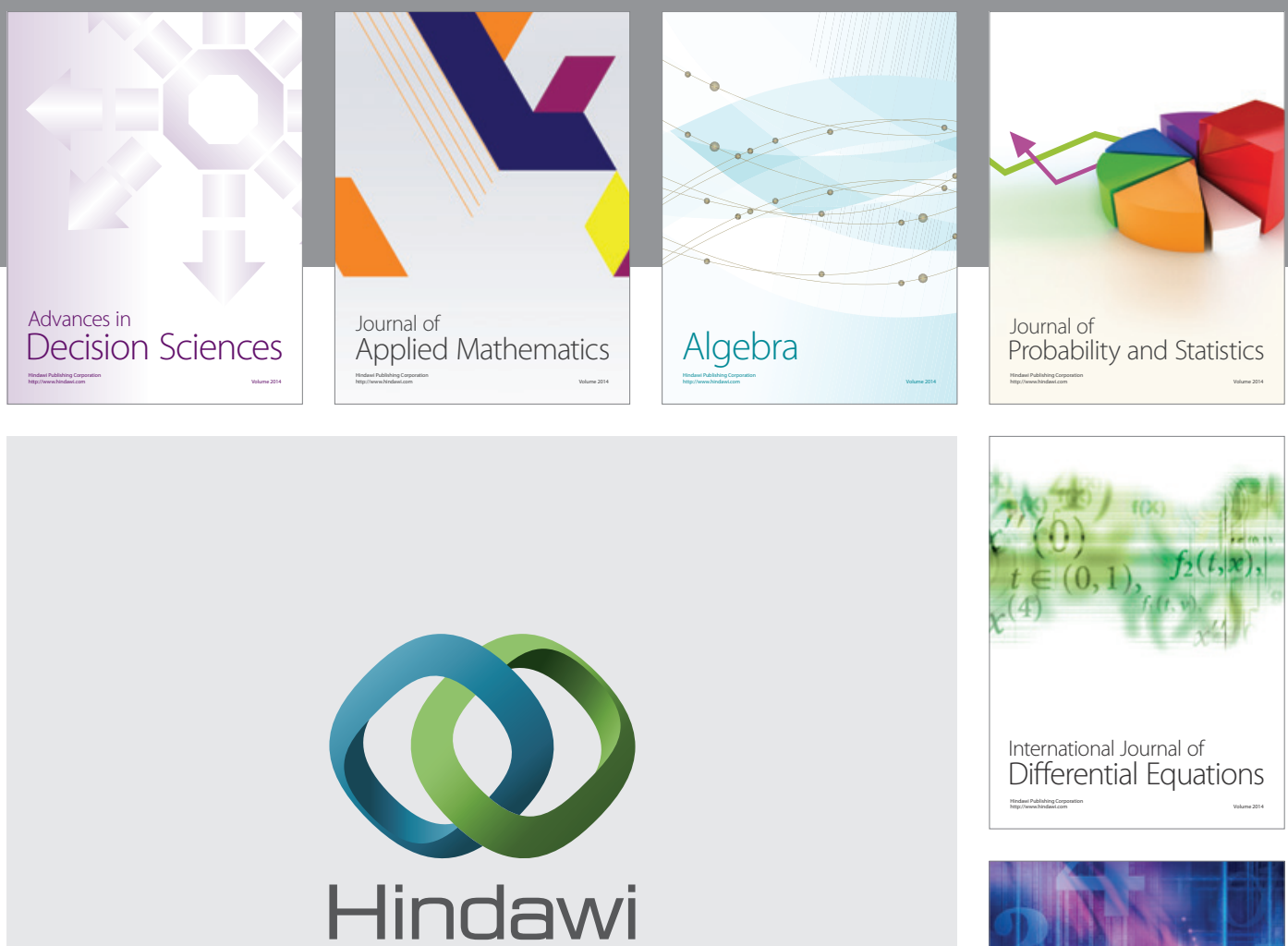

Submit your manuscripts at http://www.hindawi.com
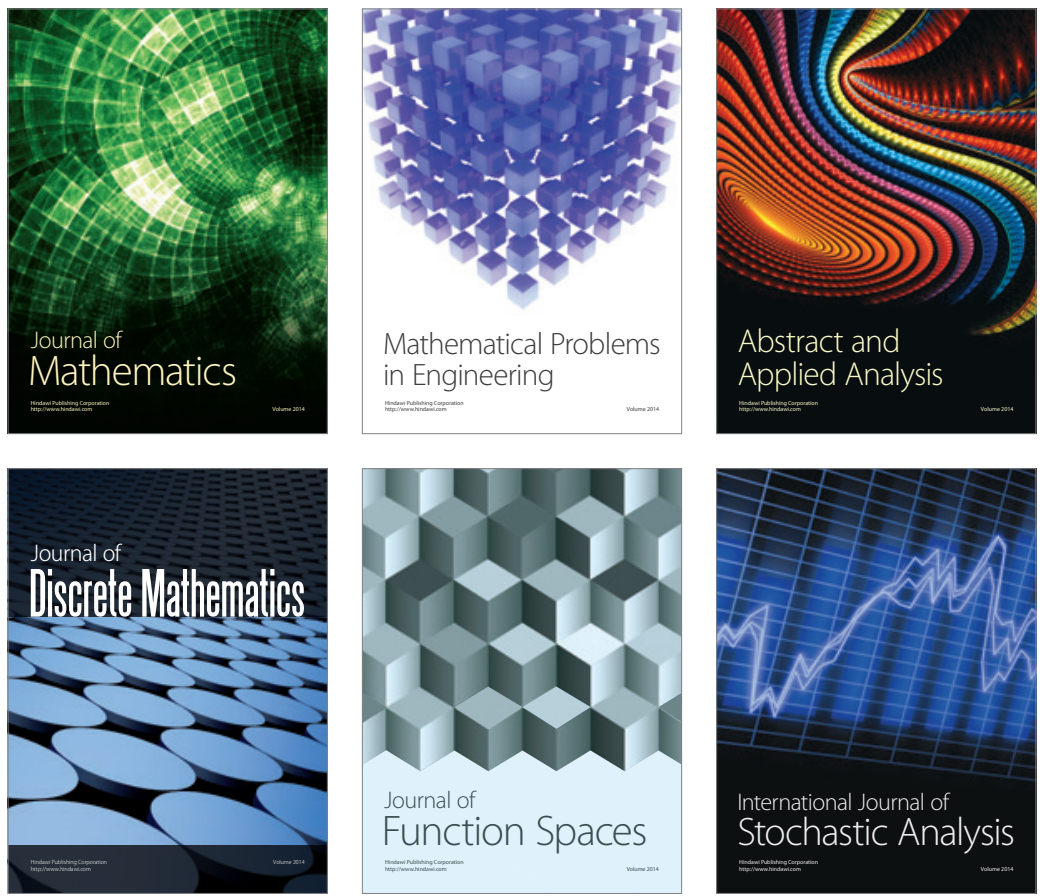

Journal of

Function Spaces

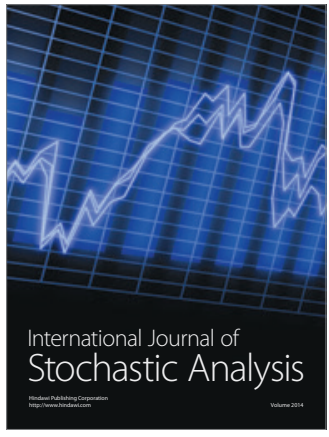

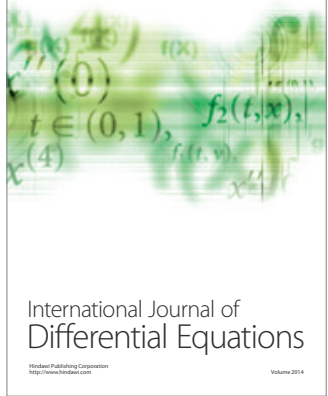
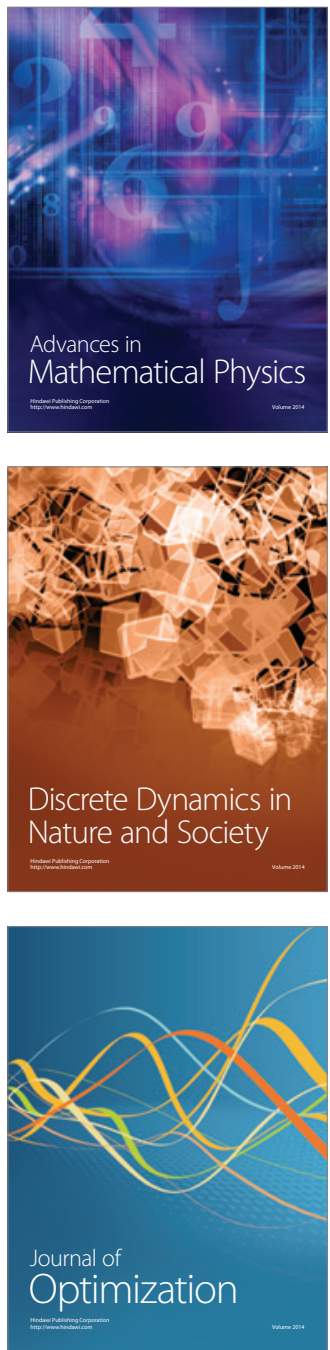